\title{
Tarif Point-of-care: document synoptique
}

\section{Anton Prant}

Président de la Direction de la Caisse des médecins
Correspondance: Anton Prantl

Caisse des médecins Steinackerstrasse 35 CH-8902 Urdorf

geschaeftsleitung@aerztekasse.ch

\section{Résumé}

Dans son rapport explicatif émis dans le cadre de la consultation sur la liste des analyses, l'OFSP invoque, pour motiver la révision de ladite liste, les progrès remarquables accomplis depuis 1994 et les changements futurs (!) qui se profilent déjà à l'horizon, tels que l'internationalisation, le progrès technologique et le comportement d'acquisition des grands laboratoires.

En outre, l'OFSP stipule que le prix des analyses doit être identique quel que soit le lieu de la fourniture de la prestation, mais dans le même souffle, il prévoit la perception par les grands laboratoires d'une taxe de base de 24 francs par demande d'analyse et la perception d'une taxe de «diagnostic en présence du patient» de 8 francs par les laboratoires de cabinet. Cette incohérence par rapport aux règles d'économie d'entreprise bouscule d'emblée toute la structure tarifaire: si au lieu d'être réalisée en laboratoire de cabinet, comme c'était généralement le cas, une analyse unique est confiée à un grand laboratoire, cette analyse coûte à l'assuré non plus 12.60 francs, mais, précisément à cause de la taxe de base, 29 francs - ce qui représente une hausse copieuse de $230 \%$, sans parler de l'absurdité écologique qui consiste à «trimballer» les échantillons d'un bout à l'autre de la Suisse.

Les rémunérations ainsi calculées (taxe de présence comprise) entraînent des baisses de prix de $30 \%$ qu'un laboratoire de cabinet typique n'a pas les moyens de supporter.

Il s'en est suivi la nécessité de développer un mode de calcul basé sur les valeurs de référence du «diagnostic en présence du patient». Cette opération n'a rien de sorcier, compte tenu de l'état toujours impeccable des données chez les médecins en pratique indépendante; l'intégration des analyses spécialement facturées au tarif du «diagnostic en présence du patient» dans la liste des analyses ne poserait aucun problème, car celle-ci connaît d'ores et déjà plusieurs segmentations de ce type.

Les répercussions du tarif Point-of-care (tarif POC) se maintiennent dans d'étroites limites, comme le montre le calcul de vérification, qui dénote une différence d'environ $3 \%$ par rapport au tarif actuel: un résultat fortuit mais heureux, si l'on considère les différences de prix tout de même considérables générées par les méthodes de calcul applicables en économie d'entreprise entre les différentes catégories d'analyses.

\section{Situation initiale}

Plusieurs désaccords apparus dans le cadre de la révision de la liste des analyses ont conduit la FMH à examiner la situation des laboratoires de cabinet:

\section{Base de la révision}

Dans son rapport explicatif émis dans le cadre de la consultation sur la liste des analyses, l'OFSP invoque, pour motiver la révision, les progrès remarquables accomplis depuis 1994 et les changements futurs (!) qui se profilent déjà à l'horizon, tels que l'internationalisation, le progrès technique et le comportement d'acquisition des grands laboratoires.

Si ces faits peuvent effectivement s'appliquer aux grands laboratoires conçus pour une production de masse, le laboratoire de cabinet typique n'est, par sa nature, pas en mesure de réaliser des économies en rationalisant. Une restructuration profonde du laboratoire de cabinet n'est envisageable ni au niveau des appareils, ni pour l'achat des réactifs.

\section{Une analyse $=$ un prix}

L'OFSP ne démord pas du point de vue que pour une analyse donnée, il ne peut exister qu'une seule méthode de calcul, et partant, un seul prix. Or, l'OFSP se départit de l'exigence qu'il a luimême posée lorsqu'il fixe une taxe de base spécifique au prestataire, à percevoir en sus du tarif de l'analyse. Il en résulte pour l'assuré des prix qui diffèrent selon le lieu où la prestation est réalisée, et qui seront typiquement plus élevés pour les analyses qui, au lieu d'être effectuées comme aujourd'hui en laboratoire de cabinet, seraient confiées demain à de grands laboratoires.

Illustrons ces propos par l'exemple de calcul d'une prestation réalisée le plus souvent en laboratoire de cabinet: un dosage de CRP en laboratoire de cabinet coûte aujourd'hui 10,80 francs. Selon les calculs de l'OFSP, cette analyse ne coûtera plus que 9 francs, mais elle sera renchérie de la taxe de base de 24 francs si elle est effectuée dans un grand laboratoire!

\section{Des différences énormes \\ dans les bases de calcul}

D'après nos connaissances actuelles, l'OFSP calcule le prix des analyses couramment effectuées par les laboratoires de cabinet sur la base d'un modèle conçu pour les grands laboratoires et pré- 
tend tenir équitablement compte des spécificités du laboratoire de cabinet en lui accordant la perception d'une taxe de diagnostic «en présence du patient» pour chaque séance de laboratoire. Or, il en résulte une baisse de $20-30 \%$ du montant des remboursements pour le laboratoire de cabinet. Le détail piquant est que cette même taxe de présence est plus de 3 fois plus élevée pour les grands laboratoires!

Les déclarations de l'OFSP pour justifier les montants de ces forfaits restent assez vagues; l'OFSP affirme avoir déterminé ces montants à partir des calculs d'une analyse des flux de production (Workflow Analysis). Pourtant, les auteurs de cette analyse ont clairement déclaré (y compris à l'OFSP) que les montants calculés représentaient une contribution à la couverture des frais et non un décompte total des coûts, et que par conséquent ces chiffres étaient utilisés par l'OFSP à mauvais escient.

\section{Une taxe forfaitaire est injustifiable d'un point de vue de gestion d'entreprise}

Une taxe forfaitaire par séance n'est acceptable que si elle est modique par rapport à la prestation productive, si elle s'applique à un mélange homogène et si elle est utilisée uniquement pour des indemnisations proportionnelles à leur objet. Aucun de ces critères ne s'applique à la taxe prévue de diagnostic «en présence du patient»:

- La taxe de diagnostic «en présence du patient» dépasse de plus du double le prix de la prestation proprement dite dans le cas du status urinaire, des analyses de chimie clinique et de la sédimentation globulaire.

- La composition et le nombre des analyses sont extrêmement variables dans le laboratoire de cabinet; le plus souvent, une séance comprend une ou deux analyses, mais il y a aussi des séances où l'on mesure plus d'une dizaine de paramètres.
- La taxe de diagnostic «en présence du patient» est censée compenser le coût de chaque analyse calculé sur des bases inapplicables au laboratoire de cabinet, mais en fait, elle est définie "par séance», ce qui constitue un cas typique d'application non proportionnelle d'un forfait!

\section{Une solution: la stratégie Point-of-care}

Avec la stratégie POC, les médecins proposent non seulement un mode de tarification conforme à la loi, mais mettent aussi clairement l'accent sur la fonction première du laboratoire de cabinet, à savoir la fourniture d'un résultat d'analyse immédiatement disponible et intégrable dans la consultation en cours. Plus précisément:

\section{La stratégie se limite à un ensemble d'analyses bien défini}

La tarification POC ne concerne que des analyses directement utilisables pendant la consultation en cours et donc susceptibles d'influencer le résultat du traitement. Le tarif POC comprend actuellement env. 30 positions, ce qui est fort modeste par rapport aux bien plus de mille positions que compte la liste des analyses. Les positions POC doivent pouvoir être facturées par tous les prestataires qui tiennent un laboratoire de diagnostic «en présence du patient», donc également par les services hospitaliers tributaires de ce type de diagnostic.

\section{Calcul spécifique en fonction des données du laboratoire de cabinet}

Les données actuelles du laboratoire de cabinet sont utilisées pour le calcul des positions POC. Celles-ci comprennent, outre les petits appareils spécifiquement utilisés pour le diagnostic «en présence du patient», les prix des réactifs tels que pratiqués sur le marché pour les quantités vendues aux laboratoires de cabinet et non aux grands

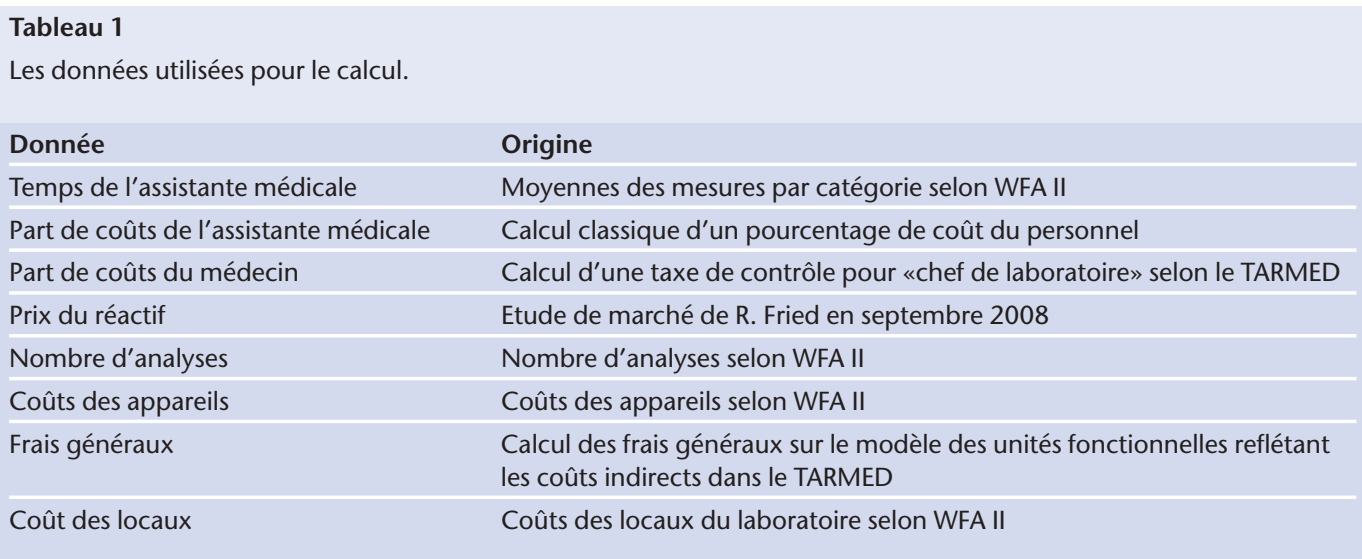


laboratoires, ainsi que le temps passé à la détermination d'un résultat par une assistante médicale dûment formée.

\section{Ni forfaits, ni taxes de base}

Comme nous l'avons déjà expliqué dans la critique du projet de l'OFSP, les forfaits et les taxes de base sont inadmissibles en raison de la polyvalence du laboratoire de cabinet.

\section{La tarification Point-of-care}

Extrêmement transparent dans sa structure, le modèle de calcul est facile à comprendre et aisément adaptable. Contrairement au secret qui en-

\begin{tabular}{ll}
$\begin{array}{l}\text { Tableau } \mathbf{2} \\
\text { Les formules. }\end{array}$ \\
\begin{tabular}{ll} 
Elément de résultat & Calcul \\
Assistante médicale & [liste_positions.temps_ass_méd] $\times[$ val_réf.coût_ass_méd] \\
\hline Réactif & {$[$ liste_positions.prix_réactif] $(\times[$ [facteur_quantité] $)$} \\
\hline Frais fixes & [catégorie.coût_appareils] / [catégorie.nombre_analyses] + \\
[val_réf.coût_locaux] $/ \Sigma[$ [catégorie.nombre_analyses]
\end{tabular} \\
\hline Médecin & [val_réf.coût_médecin] \\
\hline Frais généraux & [liste_positions.temps_ass_méd] $\times$ [val_réf. part_frais généraux] \\
\hline Prix & Ass_méd. + réactifs + frais fixes + médecin + frais généraux
\end{tabular}

Figure 1

Les répercussions du tarif $\mathrm{POC}$.

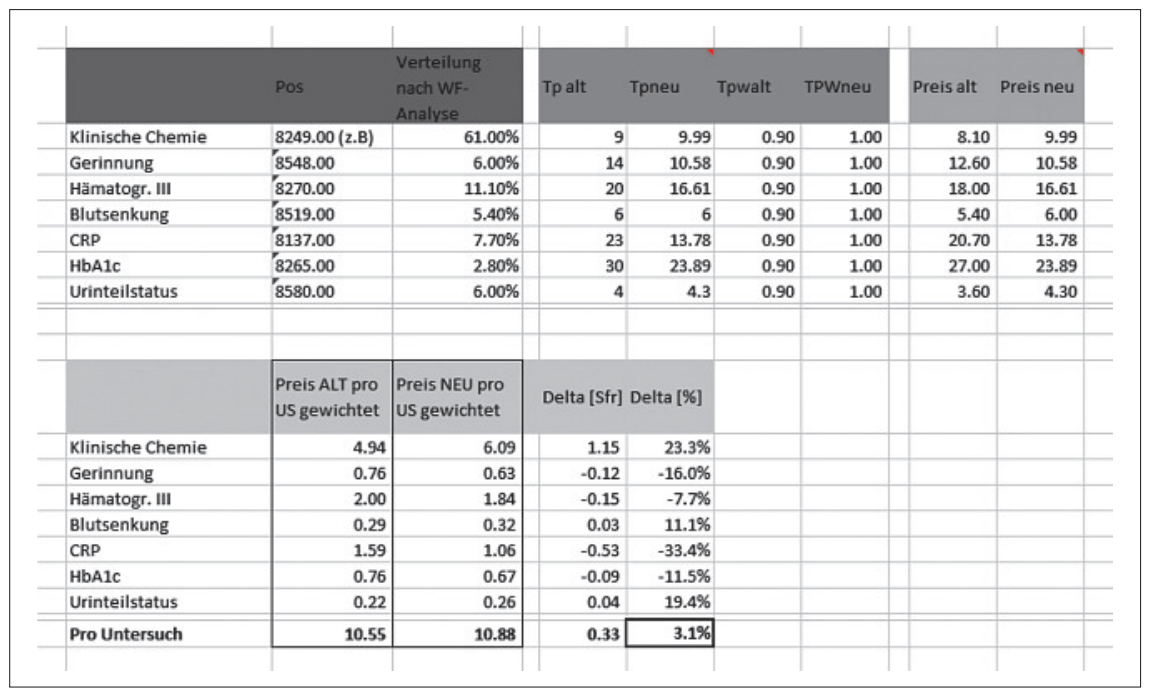

toure le modèle de l'OFSP, ce modèle-ci est public et n'a rien à cacher. Il est largement basé sur les données de l'analyse Workflow II (WFA II) effectuée en 2006, données que l'OFSP reconnaît puisqu'il les utilise pour calculer sa taxe de diagnostic «en présence du patient».

\section{Relations}

Tout le calcul peut être décrit à l'aide de trois relations:

\section{Liste des positions}

Liste des positions à calculer avec leurs variables - prix d'achat net du réactif nécessaire;

- durée de l'analyse (sans le prélèvement de l'échantillon).

\section{Liste des appareils}

Liste des appareils utilisés et de leurs variables

- coûts de chaque appareil (entretien, assurancequalité, amortissement);

- nombre d'analyses effectuées par appareil.

\section{Valeurs de référence}

Valeurs de référence du modèle de calcul

- part de coûts de l'assistante médicale;

- part de coûts du médecin;

- part des frais généraux;

- part de coûts des locaux.

\section{Bases des données}

Toutes les données utilisées pour le calcul sont récentes et s'appuient sur des bases solidement étayées:

\section{Répercussions du tarif POC}

Les répercussions du tarif POC peuvent être calculées avec précision. Comparés au tarif actuel, les prix pondérés en fonction des quantités sont supérieurs de $3,1 \%$.

Le calcul de vérification, qui compare le tarif appliqué aujourd'hui au nouveau tarif POC pondéré en fonction des quantités, montre que ce dernier n'a guère d'incidence importante $(3 \%)$ sur les coûts totaux en dépit d'importantes différences de prix entre les catégories d'analyses. Ce résultat, quoique bienvenu, n'est en aucun cas le fruit d'une intention délibérée. 